\title{
Entre métodos activos y pasivos. La educación musical en el nivel básico y superior
}

Dra. Diana Brenscheidt gen. Jost

Universidad de Sonora

diana.brenscheidt@capomo.uson.mx

\section{Introducción}

Desde inicios del siglo XX, la educación musical ha experimentado cambios fundamentales en referencia a sus métodos de enseñanza y aprendizaje. Esos aspectos se desarrollaron en buena medida, a partir de nuevos paradigmas pedagógicos que se enfocaron, entre otras cosas, en la idea de una formación integral y activa de los individuos, a diferencia de los modelos tradicionales, que adscribían al alumno un rol más bien pasivo en el proceso educativo. Hoy en día, los maestros en música pueden recurrir gracias a ello, a una variedad de métodos y técnicas musicales educativas, cuyo conocimiento y aplicación varía según lugar y nivel de escolaridad (educación básica o superior). Con el fin de brindar un conocimiento más exacto de los propósitos que persiguen algunas corrientes dominantes de la educación musical moderna en estos dos niveles educativos, el presente artículo da inicio elaborando una breve exposición de las tendencias pedagógicas desarrolladas a partir del siglo XX, las cuales influyeron de manera fundamental en la educación musical de nuestros días. Ello ha posibilitado un enfoque más universal que reconoce que el fenómeno musical recorre, como ha sido afirmado, "la totalidad de la vida de los seres humanos" (Frega en Hargreaves, 1998, p. 5). Con base en lo anterior, y ampliando la discusión, se investiga y describe posteriormente, cómo se han aplicado dichos modelos en la educación musical básica y superior, buscando con ello mostrar tendencias internacionales o regionales que puedan servir como base para futuras discusiones y ajustes en la educación musical en México y Latinoamérica.

\section{Paradigmas metodológicos: educación musical para todos}

Se ha propuesto, mientras que la educación musical de los siglos XVIII y XIX se basó en modelos tradicionales positivistas, enfocados en aspectos de lectoescritura y el tratamiento de la música como un objeto (Jonquera Jaramillo, 2004, p. 22), la investigación psicológica y los cambios pedagógicos fundamentales del siglo XX llevaron a entender el proceso de enseñanza musical vinculado con la educación y la formación general de las personas (véase Gaston, 1968). Esta aceptación se refleja en los métodos y conceptos de la educación musical, los cuáles experimentaron un desarrollo vertiginoso a partir del siglo XX. Autores, pedagogos y músicos como Dalcroze, Kodály, Orff, Martenot, Willems y Suzuki fundamentalmente enriquecieron el proceso de enseñanza-aprendizaje de la música, cada uno de modo distinto. Los tres primeros, se ha sugerido, "cuentan con la mayor difusión a nivel mundial y siguen teniendo importancia y vigencia aún en la actualidad" (Jonquera Jaramillo, 2004, p. 22). Según Pilar Pascual Mejía (2002), a 
los teóricos del siglo XX los unió la "idea de que la educación musical no puede ser patrimonio exclusivo de unos pocos" (p. 102). Elisa Roche Márquez (2005) señala cómo esos ideales estaban "estrechamente vinculados a todo el movimiento de renovación pedagógica que representa la Escuela Nueva y afectan tanto a la enseñanza de la música como a la formación integral de la persona" (p. 11). En sintonía con el resurgimiento de ideas humanistas en el siglo XX, Kodály expresó en los sesentas, que "la música es una parte indispensable de la cultura humana universal"; "por lo tanto", añadió, "aquellos que no poseen conocimientos musicales tienen un desarrollo intelectual imperfecto". Sin música "no existe hombre completo, integral. Por esto", advirtió, "es absolutamente natural y necesario que la música se integre en el currículo escolar." (Kodály en Roche Márquez, 2005, p. 10). Según Jonquera Jaramillo (2004), la Escuela Nueva de pedagogía tuvo como precursores a quienes iniciaron el debate de los modelos educativos durante el siglo XVIII y XIX, destacando Rousseau, Pestalozzi y Fröbel. Se dio después un nuevo impulso a finales del siglo XIX, pero sobre todo durante el siglo XX, con aportes como las de la escuela activa ( $\mathrm{p}$. 13). Educadores y filósofos de procedencia europea y norteamericana, como Adolphe Ferrière, John Dewey, Ovide Decroly, Maria Montessori y Jean Piaget, empezaron una reforma de la enseñanza, la cual se fundamentó "en la acción, en la actividad del niño" (Gadotti, 1999, p. 147) y proclamaron "la necesidad de una educación para todos, la democracia en la educación" (Hemsy de Gainza, 2004, p. 3). Aunque Jonquera Jaramillo (2004) no constata ninguna relación directa entre la Escuela Nueva y los pensadores de la educación musical del siglo XX, afirma que la Escuela Nueva "tuvo gran influencia en el ámbito de la educación musi- cal" manifestándose particularmente en sus métodos (p. 13). Esa misma opinión la comparte Violeta Hemsy de Gainza (2004), quien señala que los métodos activos pedagógicos "influencian posteriormente la educación musical” (p. 2).

Jonquera Jaramillo (2004) identifica así la base de los conceptos centrales de la educación musical del siglo XX en distintos modelos de acción didáctica, específicamente en el tradicional y en el activo. La diferencia entre ellos se manifiesta primeramente, sugiere, al considerar el papel de los actores involucrados en el proceso de enseñanza-aprendizaje, lo que se refleja en la educación musical:

En el modelo tradicional, la actividad didáctica se centra en el profesor -podríamos denominar esta característica magistrocentrismoy en los contenidos que éste transmite, mientras en el modelo activo, se atribuye gran importancia a la participación del alumno [denominado el paidocentrismo], con el fin de que él mismo comprenda la materia, realice actividades creativas, destacándose el descubrimiento y la experimentación. (p. 12f)

El cambio de un modelo tradicional a otro activo en la educación musical del siglo XX es descrito también por Pascual Mejía (2002), quien se concentra particularmente en el nivel básico. Según dicha investigadora, pedagogos y músicos como Dalcroze, Orff, Kodály, Martenot, Willems y otros, pretendían transformar "los esquemas rígidos de una enseñanza de la música basada en el solfeo y el aprendizaje de un instrumento y abrieron las puertas a una formación musical en la que 'hacer música' y 'vivir la música' era más importante que 'saber la música o teorizar sobre la música'” (pp. 16ff). Así mismo, Keith Swanwick, al estudiar la reforma fundamental que se realizó en el 
ámbito de la educación musical, enfatizó cómo los teóricos del siglo XX cambiaron la concepción "del alumno como 'heredero', pasando al alumno como 'persona que disfruta', entendiéndolo como un 'explorador' y un 'descubridor'” (p. 17). El enfoque activo, bajo la premisa de educación musical para todos, tuvo repercusiones fundamentales en el campo de la educación básica, trayendo como resultado, según Violeta Hemsy de Gainza (2004), la denominación del siglo XX como "el siglo de la iniciación musical" (p. 1).

\section{La educación musical básica}

Junto con lo anterior, se gesta a su vez el desarrollo de la investigación cognitivo-musical (véase Sarget Ros, 2003), en la cual, autores y profesionales del nivel educativo básico destacan el rol de la música en el desarrollo integral de niños y subrayan cómo la música ayuda a formar varias capacidades fundamentales, desde las auditivas, expresivas y psicomotrices, hasta las sociales, críticas y estéticas. Respecto al nivel básico, Pilar Pascual Mejía (2002) señala:

La educación musical en Primaria tiene un objetivo final que es la formación integral de la persona en todos los aspectos de su personalidad a través de la música [...]. La educación por la música colabora con el resto de las materias educativas, aunque difiere en sus propios objetivos específicos. [...] La percepción auditiva de la música (tiempo, espacio, ritmos, melodías ...) servirá de gran apoyo para el desarrollo de la inteligencia y otros aprendizajes de representaciones visuales y gráficas como la lectura o la escritura. La percepción musical y la expresión musical son las dos capacidades que debe desarrollar la enseñanza de la música en Primaria. (p. 8)
El enfoque de Pascual Mejía sobre la percepción y expresión musical para alumnos del nivel básico se basa en los diferentes métodos de la enseñanza musical, los cuales emergieron de forma innovadora durante el siglo XX y han mantenido su relevancia hasta hoy. Entre ellos destaca, generalmente subsumida bajo la denominación de 'métodos activos' -debido a su relación con los modelos de aprendizaje pedagógico-, la integración de la música con las demás artes o técnicas corporales, con el objeto de evocar un entendimiento más integral. Un ejemplo influyente de lo anterior es representado por el pedagogo y músico suizo Emile Jaques-Dalcroze (1865-1950). El enfoque didáctico de Dalcroze, conocido como "rítmica", se concentra en integrar las habilidades musicales con el movimiento corporal, teniendo como fin desarrollar las facultades artísticas de las personas. Investigando "el rol importante del movimiento corporal en la conciencia y clarificación del movimiento musical" (Del Bianco, 2007, pp. 23-24), el pedagogo inició un método didáctico multidisciplinario que combina técnicas del solfeo con el movimiento en el espacio, lo cual, en su origen, estaba pensado originalmente para adultos (Pascual Mejía, 2002, p. 100). Sin embargo, el método de Dalcroze, su combinación de música, rítmica y danza en el espacio, fue empleado en el nivel básico por su utilidad para entrenar habilidades psicomotrices y por su aplicación para el desarrollo del oído y del lenguaje musical a través del cuerpo. Pilar Pascual Mejía declara que su "mayor aplicación es en los niveles iniciales en los que se da un comienzo, una iniciación a la música" (p. 119). La investigadora concluye indicando las ventajas de la rítmica: "el principal mérito del método es el que tiende a una educación integral de la persona en la que se trabajan simultáneamente la atención, la inteligencia, la rapidez mental, la sensibilidad y el movimiento" (p. 120). Por otro 
lado, según Hemsy de Gainza (2004), Edgar Willems (1890-1978) y Maurice Martenot (1898-1980), conocidos ambos por sus contribuciones a la educación musical, desarrollaron sus métodos en relación con la obra de Dalcroze, por lo cual, incluye a ambos dentro de una mismas tradición (p. 3).

También Carl Orff (1895-1982), compo- sitor y pedagogo germano, desarrolló una propuesta didáctica que combina la música con las demás facultades humanas, incluyendo la danza y el movimiento. La propuesta teórica de Orff se basa en la semejanza estructural y mutua complementación entre el lenguaje verbal y musical (Conde Caveda et al., 2002, p. 37). Según Orff, "hablar es hacer música" (ibíd.), por el motivo de que el lenguaje hablado, con

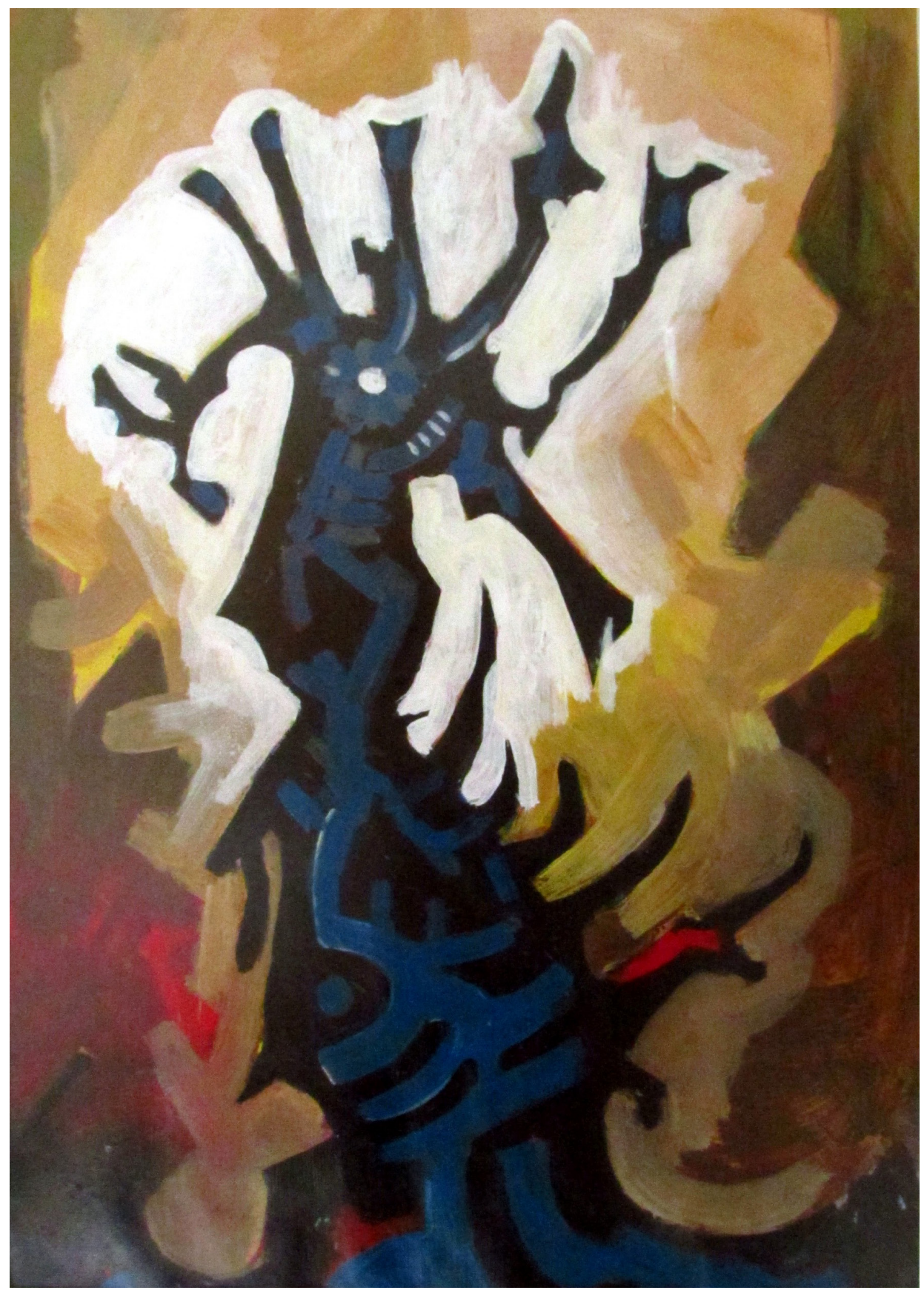


aspecto rítmico y expresivo, nos lleva al canto, que se entiende como la continuación del lenguaje. La obra didáctica de Orff, el Schulwerk (u 'obra escolar' en castellano), se concentró específicamente en los infantes y se resume en cinco libros, bajo el título de Música para niños, incluyendo varias piezas instrumentales y canciones (López Ibor, 2007, p. 73). Una propuesta fundamental de su método es el uso de instrumentos hechos en tamaño y materiales específicos para niños, con la finalidad de tocar música elemental en grupos (Lange, 2005, p. 9). Hemsy de Gainza (2004) clasifica el aporte de Orff, junto con los del húngaro Zoltán Kodály (1882-1967) y del japonés Shinichi Suzuki (1898-1998), bajo la de nominación de "métodos instrumentales, equiparando así a Kodály, quien se concentró en la voz y aspectos de lectoescritura musical, con Suzuki, quien desarrolló por su parte un método para la enseñanza temprana del violín (p. 3). También acentúa la importancia de la recepción de Kodály en Argentina y otros países de Latinoamérica para aprender "a valorar el folclore, objeto imprescindible de la educación musical" en el nivel básico (2003, p. 5).

Mención aparte requieren por último, autores como el canadiense Murray Schafer (nacido 1933) y los británicos John Paynter (1931-2010) y George Self, integrantes, según Hemsy de Gainza, de la llamada "generación de los compositores". Dicha autora los valora por su habilidad de compartir su creatividad con los alumnos, introduciendo por la primera vez la música contemporánea en las aulas (2004, p. 6).

La presentación de los aportes más significativos de la educación musical del siglo XX que hasta aquí se ha realizado, tuvo como fin describir la multiplicidad de propósitos ofrecidos por los distintos pensadores y músicos en el marco de la educación básica. Sin embargo, no importando qué tanto difieran éstos entre sí, todos coinciden en involucrar al alumno "como participante activo de su propio aprendizaje" (Jonquera Jaramillo, 2004, p. 13), así como también en concebir la música como una parte indispensable en la educación integral de la persona. Por consiguiente, Pilar Pascual Mejía (2002) ubica la base de la didáctica musical para primaria en los métodos activos y afirma: "Las principales metodologías pedagógico-musicales entienden la educación musical con métodos activos y no intelectivos, en los que aprender música es sinónimo de hacer música" (p. 14). Cada uno de los enfoques aquí presentados, pensados inicialmente para niños o no, dejó su huella en la concepción y ejecución de clases en la educación musical básica o inicial. Aunque originados en Europa, su aplicación en los países de Latinoamérica ha sido documentada (Hemsy de Gainza, 2004). Sin embargo, como analiza y expone Jonquera Jaramillo (2004), cada método tiene distintos enfoques y por eso existen limitaciones que complican la situación de los maestros de música en las escuelas, al momento de escoger uno, así: [...] ninguno de los conceptos expuestos agota por sí solo las temáticas de la educación musical, hecho determinante para considerar insuficiente cada uno separadamente como medio para enfrentar las tareas de la educación musical escolar en el aula. Por ello, es necesaria una visión amplia, un conocimiento de un vasto número de propuestas, no necesariamente articuladas en un concepto específico, pero que si puedan constituir un repertorio al cual recurrir en las muy variadas situaciones que se le presentan al profesor en el aula. (p. 51)

Hemsy de Gainza (2000) confirma y valora la multiplicidad de opciones del maestro o profesor de música y enfatiza la necesidad "de adoptar, en cada caso particular, en los procesos de enseñanza-aprendizaje, criterios pedagógicos adecuados: al objeto de conocimiento, a las necesidades individuales y sociales de alumnos y profesores, y a la coyuntura 
educativa y social" (p. 7). Faltaría agregar finalmente, también a las necesidades culturales a las que se enfrentan los maestros de música en el nivel básico. Según dicha investigadora, los retos como los del multiculturalismo ya entraron a la educación musical en los ochentas y noventas y con ellos el reconocimiento de "la necesidad de dar a los alumnos una formación amplia que, sin descuidar la propia identidad, permita integrar otras músicas, otras culturas" (2004, p. 8). En Alemania, por ejemplo, país que aloja un número significativo de migrantes y que, por efecto de la Unión Europea y su ubicación geográfica en el centro del Viejo Continente, experimenta los efectos del multiculturalismo de manera directa, el tema de una pedagogía musical intercultural ha entrado al debate educativo desde hace años, reflejándose en el currículo escolar básico (véase, por ejemplo, Rodríguez-Quiles y García \& Jank, 2009).

En correspondencia con los autores antes mencionados, la educación musical básica deberá fundamentarse entonces en la formación integral y personal del alumno como agente principal del proceso educativo, desarrollando sus habilidades musicales (auditivas, rítmicas, instrumentales, etc.) a través de los distintos métodos ofrecidos por los teóricos y pensadores de la educación musical del siglo XX, empleados hasta hoy pero considerando las necesidades individuales, sociales y (multi)culturales de los alumnos y maestros de manera flexible. A lo anterior se suma como reto la formación de profesores especializados en música, ya que, como documenta Luis Alfonso Estrada (2001) para la UNESCO, en un informe sobre la educación básica en México:

En la mayoría de las escuelas primarias del país, los profesores que atienden a los grupos en todas las materias (Español, Matemáticas, Historia, Geografía, etc.) son los encargados de llevar a cabo las actividades artísticas. En muy pocas escuelas, en un número extremadamente reducido para todo el país, hay escuelas Primarias, en su mayoría privadas, que cuentan con profesores de música (p. 3). [Cita textual del informe de Luis Alfonso Estrada]

Sin perder de vista este escenario, se describirán a continuación los elementos mínimos indispensables que permitan comprender los propósitos de la educación en música en el nivel superior.

\section{Educación musical superior}

Considerando que las principales repercusiones de las reformas en educación musical del siglo $\mathrm{XX}$ se suscitaron en el nivel básico, la situación en el nivel superior, tanto en Europa como en Latinoamérica, siguió operando en la lógica de los modelos tradicionales de enseñanza-aprendizaje; es decir, bajo un modelo educativo enfocado en el profesor y en la ejecución de obras maestras por parte del alumno. Jonquera Jaramillo (2004) menciona que, en el contexto de la educación superior en Europa, específicamente en las clases magistrales universitarias, el modelo tradicional todavía conoce una amplia utilización, no obstante las reformas pedagógicas del siglo pasado (p. 12). Con una investigación desarrollada en 2004, Violeta Hemsy de Gainza denunció que: "el nivel de la formación musical especializada o superior, como ya lo expresamos, continua desactualizado: la mayor parte de las reformas educativo-musicales del siglo XX sucedieron en el campo de la educación general y de la educación musical inicial, mientras los conservatorios y las universidades permanecen al margen de los cambios" (p. 9).

Tiempo atrás, la especialista había demandado la actualización del nivel superior de educación musical y el aumento de investigación en dicho campo, con el fin de formar profesionales de la música aptos para elevar la calidad de la enseñanza (2000, p. 7). En su visión, sólo un reduci- 
do número de países de la Europa del norte pudieron sortear los problemas de falta de actualización en la educación musical superior, algo que adjudicó al mayor valor que por lo general, en sus respectivas culturas, ortorgaban a la formación musical (2004, p. 10). Para Héctor Pérez López (2006), por ejemplo, en el nivel superior existen dos modalidades dominantes de estudios en música: uno, que tiene como propósito el performance, característico de la escuela estadounidense, y "que supone una especialización concentrada exclusivamente en el ulterior desarrollo o avance en las destrezas propias de la interpretación, adquirida en los estudios de grado y/o máster", mientras la otra, "ampliamente extendida en Australia y dominante en los países europeos que poseen el tercer ciclo en música", persigue "una visión relativamente nueva, que busca una aportación neta de las destrezas intelectuales propias de la investigación en el ámbito artístico" (p. 51). Según Mari Carmen Gómez Muntané (2006), es este "doble perfil" el que posibilita mejores ofertas de trabajo para egresados de las universidades y conservatorios de música en países como España. "Si hasta no hace mucho el desarrollo de uno de los dos perfiles, el que conjuga el conocimiento musical con el humanístico, era el priorizado, la tendencia actual", afirma, "tiende incluso a equilibrar ambos perfiles, la de músico y la de musicólogo, favoreciendo la unión entre práctica e investigación musical bajo el mismo perfil" (p. 80). Un ejemplo de este propósito educativo en música se puede observar en la Universidad de Indiana, en los Estados Unidos, cuyo departamento de música es reconocido por su carácter integral (véase Logan, 2000). Entre los propósitos educativos que enarbola destacan: "1. Preparar a los estudiantes para desarrollar carreras como intérpretes, compositores, profesores, maestros, etc; 2 . Proporcionar a estudiantes de música la oportunidad de desa- rrollar sus conocimientos, entendimiento y capacidades en todos los aspectos de la música, en niveles adecuados a sus necesidades e intereses; 3 . Ampliar y profundizar el conocimiento y entendimiento de todos los aspectos de la música a través de la investigación y las publicaciones; y 4. Enriquecer las vidas de los estudiantes, la comunidad, la nación y el mundo con interpretaciones de una amplia variedad de música" (Gutiérrez Barrenechea y Piñero Gil, 2007, p. 89). Aunque los propósitos no mencionan directamente la aplicación de métodos activos, el segundo aspecto enuncia la base de un modelo pedagógico paidocéntrico (véase Jonquera Jaramillo, 2004, pp. 12f) enfocado en las necesidades y la creatividad del estudiante.

Como se sugiere en el último propósito enumerado, la variedad de la música existente y accesible al individuo, en lo que Manuel Castells ha llamado "sociedad del conocimiento", se multiplica con el empleo masivo de medios de comunicación y bajo el impulso del multiculturalismo, representando un reto al entendimiento, tanto del alumno como del profesor de música. La investigación musical ha permitido afirmar que "un aprendizaje basado en un repertorio musical 'plural' contribuye al desarrollo de una consciencia multicultural asentada en la comprensión mutua y en la tolerancia, promueve un mayor entendimiento y aceptación entre las personas de diferentes culturas, favorece una mentalidad más abierta y ayuda a erradicar prejuicios raciales y generacionales" (Giraldez, 1997, p. 8).

Para desarrollar un perfil musical que ensamble capacidades ejecutivas o instrumentales como también investigativas, abarcando las músicas de diferentes épocas, culturas y grupos sociales, se requiere de instituciones de educación musical superior más integradoras. Bajo esa premisa fue creada la "Hochschule für Musik, Theater und Medien" (Escuela superior para música, teatro y medios de 
comunicación) en Hannover, Alemania, que ha desarrollado programas superiores interdisciplinarios que combinan estudios instrumentales de las diferentes épocas y grupos sociales (de la música de la Edad Media y del Renacimiento hasta la música contemporánea, el jazz y la música popular) con disciplinas como Musicología, Sociología Musical, Etnomusicología, Musicoterapia, Pedagogía Musical, Teatro y el empleo de tecnologías de la información y la comunicación.

\section{Conclusión}

Aunque los propósitos formativos de la educación musical en los niveles básico y superior han sido considerados aquí de forma independiente, debido a su estrecha y compleja relación requieren considerarse también de forma conjunta. En un mundo donde lo local está cada vez más interconectado, como sugiere Marshall McLuhan con su concepto de "aldea global", se tiene la tendencia a hacer equivalentes los distintos sistemas educativos, situación que no es privativa de un solo nivel educativo ni de una región del planeta. La necesidad de vincular todos los niveles de la educación, desde el básico al superior, ha sido constatada en un estudio dirigido por la pedagoga gallega López de la Calle Sampedro (2007), quien al referirse a la educación musical advirtió que:

La idea de integrarnos en el Espacio Europeo de Educación Superior nos exige el esfuerzo de mirarnos hacia otros contextos educativos europeos. Conocer y comparar cómo se desarrolla la docencia de la música en centros educativos de educación infantil de otros países cobra, a nuestro juicio, una especial importancia en estos momentos (p. 111).

Se desea agregar finalmente que, para poner remedio a la "ausencia de la reflexión pedagógica en el ámbito de la educación musical" (Abel-Struth en Jonquera Jaramillo, 2004, p. 23), se hace necesario un sistema de educación musical basado en modelos pedagógicos "activos", centrándose en el desarrollo integral de las capacidades del alumno (psicomotrices, auditivas, expresivas, creativas, críticas, etc.). Además, un sistema educativo musical que empiece desde el nivel básico, el cual permita a la postre una educación musical superior más amplia, la cual debe mantener las capacidades ejecutivas y creativas, a la vez que incorpore el conocimiento investigativo (musicológico, pedagógico y preferentemente cultural) con la finalidad de difundir y enriquecer conocimientos musicales en la sociedad global para las futuras generaciones. Caminar en ese sentido y continuar en la investigación respectiva desde varios referentes culturales (véase McPherson \& Welch, 2012), sería innovar en los propósitos de una educación musical integrada y acorde con los requerimientos del siglo XXI.

\footnotetext{
Bibliografía

Conde Caveda, José Luis et al. (2002). Las Canciones Motrices II. Metodología para el Desarrollo de las Habilidades Motrices en Educación Infantil y Primaria a través de la Música. Barcelona: INDE.

Del Bianco, Silvia (2007). "Jaques-Dalcroze". En: M. Díaz \& A. Giráldez (coords.). Aportaciones teóricas y metodologías a la educación musical. Barcelona: GRAÓ. 23-32.
} 
Estrada, Luis Alfonso (2001). "Informe que se presenta en el marco del Programa para la Promoción de la Educación Artística a nivel escolar: Primaria y Secundaria". UNESCO Regional Meeting of Experts on Arts Educación at school level in Latin America and the Carribean. Brazil. 1-26.

Gadotti, Moacir (1999). Historia de las Ideas Pedagógicas. México: Siglo XXI.

Gaston, E. Thayer (1968). Music in Therapy. New York: Macmillan.

Giraldez, Andrea (1997). "Educación musical desde una perspectiva multicultural: Diversas aproximaciones". Revista Transcultural de Música / TRANS Iberia 1. 117.

Gómez Muntané, Mari Carmen (2006). “Campos, temas y metodologías de la investigación relacionadas con las artes: Algunas reflexiones sobre el caso de la Musicología". En: Idem. Bases para un debate sobre investigación artística, Madrid: Omagraf. 73-86.

Gutiérrez Barrenechea, María del Mar \& Carmen Cecilia Piñero Gil (2007). La formación de intérpretes profesionales en los conservatorios en el marco de la reforma educativa: Madrid como paradigma. Madrid: Ministerio de Educación.

Hargreaves, David J. (1998). Música y desarrollo psicológico. Barcelona: GRAÓ.

Hemsy de Gainza, Violeta (2004). "La Educación Musical en el siglo XX". Revista Musical Chilena LVIII, no. 201. 1-13.

---- (2003). "La Educación Musical entre dos siglos". Universidad de San Andrés, [sin paginación].

----- (2000). “Problemática actual y perspectivas de la educación musical para el siglo XXI". Primer Seminario y Taller de Educación Musical. Lima, Perú. 1-11.

Jonquera Jaramillo, María Cecilia (2004). "Métodos históricos o activos en educación musical", Revista electrónica de LEEME no. 14. 1-55.

Lange, Diane M. (2005). Together in Harmony. Combining Orff Schulwerk and Music Learning Theory. Chicago: GIA.

Logan, G.M. (2000). The Indiana University School of Music: A History. Bloomington: Indiana University Press.

López de la Calle Sampedro, María Ángeles (2007). La música en centros de educación infantil 3-6 años de Galicia e Inglaterra. Un estudio de su presencia y de las prácticas educativas (Tesis doctoral). Santiago: Universidad de Santiago de Compostela.

López Ibor, Sofía (2007). “Carl Orff”. En: M. Díaz \& A. Giráldez (coord.). Aportaciones teóricas y metodológicas a la educación musical. Barcelona: GRAÓ. 71-77.

McPherson, Gary E. \& Graham F. Welch (2012). The Oxford Handbook of Music Education. Vol. 1. New York: Oxford University Press.

Pascual Mejía, Pilar (2002). Didáctica de la Música para Primaria. Madrid: Pearson Educación.

Pérez López, Héctor Julio (2006). "Investigación y práctica musical: el horizonte del doctorado en música en Europa”. En: M. C. Gómez Muntané (ed.). Bases para un debate sobre investigación artística. Madrid: Omagraf. 51-73.

Roche Márquez, Elisa (2005). “La educación musical escolar: análisis histórico y valoración del currículo actual”. En: La Dimensión Humanística de la Música: Reflexiones y Modelos Didácticos. Ministerio de Educación y Ciencia. 9-24.

Rodríguez-Quiles y García, José A. \& Birgit Jank (2009). Perspektiven einer Interkulturellen Musikpädagogik. Potsdam: Universitätsverlag Potsdam. 
Sarget Ros, María Ángeles (2003). “La música en la educación infantil: Estrategias cognitivos-musicales". Ensayos. Revista de la Facultad de Educación de Albacete, no. 18. 197-209.

Swanwick, Keith (2000). Música, pensamiento y educación. Madrid: Morata.

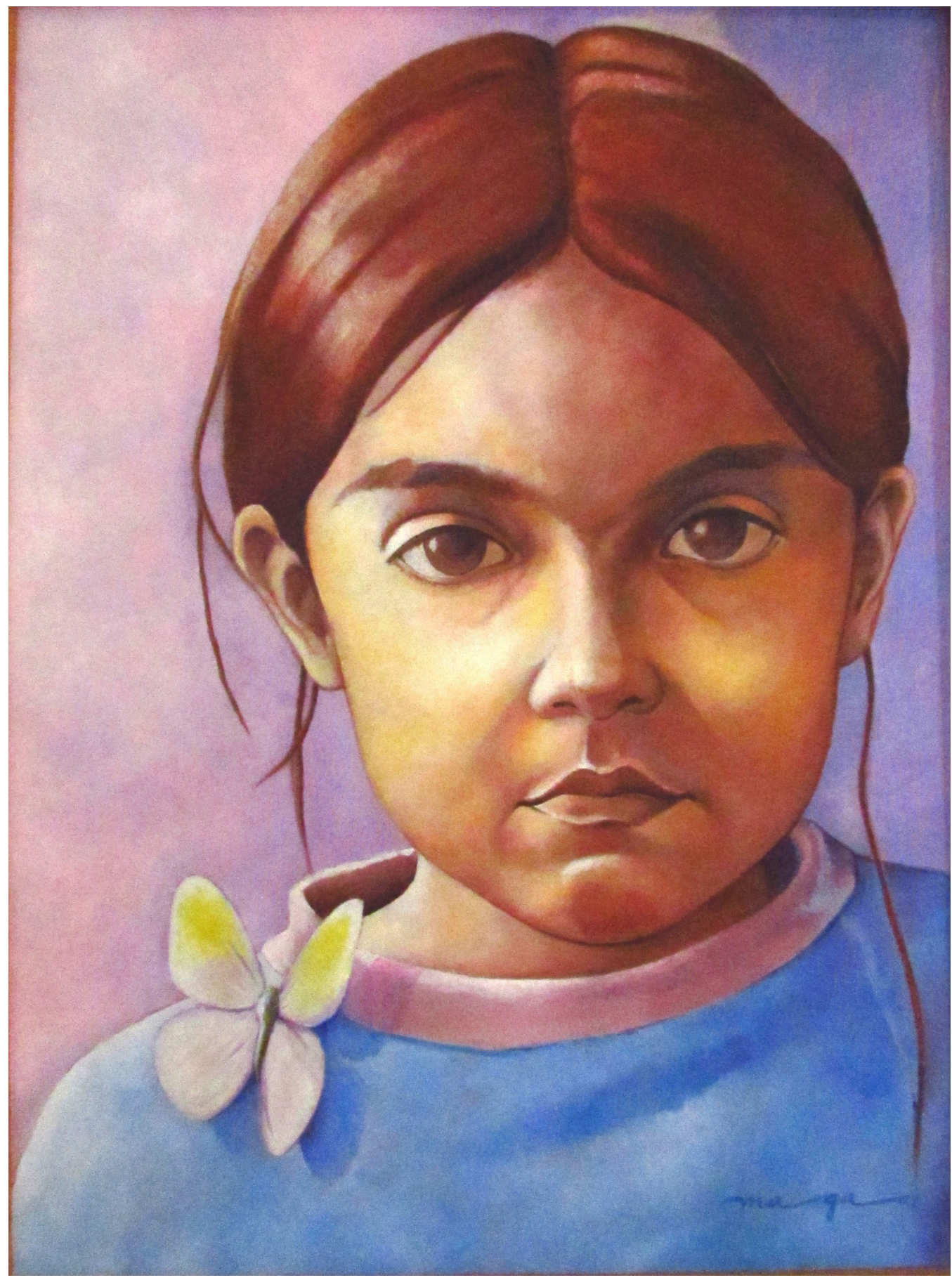

